\title{
Modélisation statistique du vent Influence du changement climatique sur le transport éolien
}

\author{
Etienne Guerber ${ }^{1,2,3}$, Gonéri Le Cozannet ${ }^{1}$, Rodrigo Pedreros ${ }^{1}$ \\ ${ }^{1}$ BRGM, Service Aménagement et Risques Naturels, 3 av. C. Guillemin, \\ BP 36009, 45060 Orléans cedex 2 \\ ${ }^{2}$ ENSTA, 32 bd Victor, 75739 Paris cedex 15 \\ ${ }^{3}$ Université Pierre et Marie Curie, 4 place Jussieu, 75252 Paris cedex 05 \\ etienne.guerber@ensta.org,g.lecozannet@brgm.fr,r.pedreros@brgm.fr
}

\section{Résumé :}

Un modèle statistique permettant de générer des séries temporelles de vent est calibré sur des données locales au large de Dunkerque (Nord-Pas-de-Calais). Ce modèle statistique est modifié pour simuler un changement du climat de vent local. Les séries temporelles ainsi générées sont ensuite utilisées pour quantifier la sensibilité du transport éolien de sédiments en zone littorale à une modification du régime des tempêtes hivernales.

\begin{abstract}
:
A statistical model able to generate wind time series is calibrated on local wind data off Dunkerque (Nord-Pas-de-Calais). This statistical model is modified to simulate local changes of the wind climate. These time series are then used to quantify the sensibility of aeolian transport of sediments on costal areas in response to a modification of the regime of winter storms.
\end{abstract}

\section{Mots-clés :}

Modélisation statistique - vent - transport éolien - tempête - changement climatique.

\section{$1 \quad$ Introduction}

La modélisation de l'évolution des littoraux en réponse aux changements climatiques requiert l'usage de forçages réalistes. Une première approche peut consister à utiliser des séries temporelles issues de simulations de modèles climatiques régionaux. Cependant, le manque de robustesse des champs de vents en sortie de ces modèles (RAÏSENEN et al., 2003) limite leur utilisation à une échelle locale. 
Comme alternative, une approche consistant à générer des séries temporelles représentatives d'un climat de vent local a été mise en œuvre par MONBET et al., (2006), afin de calculer le transport sédimentaire sur une côte idéalisée soumise à une mer de vent créée par ce forçage éolien. Nous appliquons un modèle similaire pour quantifier l'activité du transport sédimentaire éolien dans un climat actuel, puis dans un climat perturbé sur un site idéalisé inspiré de la côte sableuse de la région de Dunkerque.

Nous utilisons des données de vent de la bouée Westhinder [51.39 $\left.\mathrm{N} 2.44^{\circ} \mathrm{E}\right]$ fournies au Laboratoire d'Océanologie et de Géosciences (LOG, UMR 8187, Equipe Dynamique des Littoraux) par le VLIZ (Vlaam Instituut voor de Zee). La boîte à outils METIS (MONBET \& AILLIOT, 2005) a été utilisée pour calibrer un modèle autorégressif à changements de régimes markoviens d'ordre 1. Enfin, nous avons perturbé les paramètres de ce modèle afin de simuler des tempêtes plus fréquentes ou plus intenses, et nous quantifions l'activité résultante du transport sédimentaire éolien au moyen de plusieurs formules de transport.

\section{Données et période d'étude}

Les données de vent de la bouée Westhinder s'étalent du $1^{\text {er }}$ Janvier 1997 au 31 Aout 2007 et donnent la vitesse du vent et sa direction, avec un pas de temps de $10 \mathrm{~min}$.

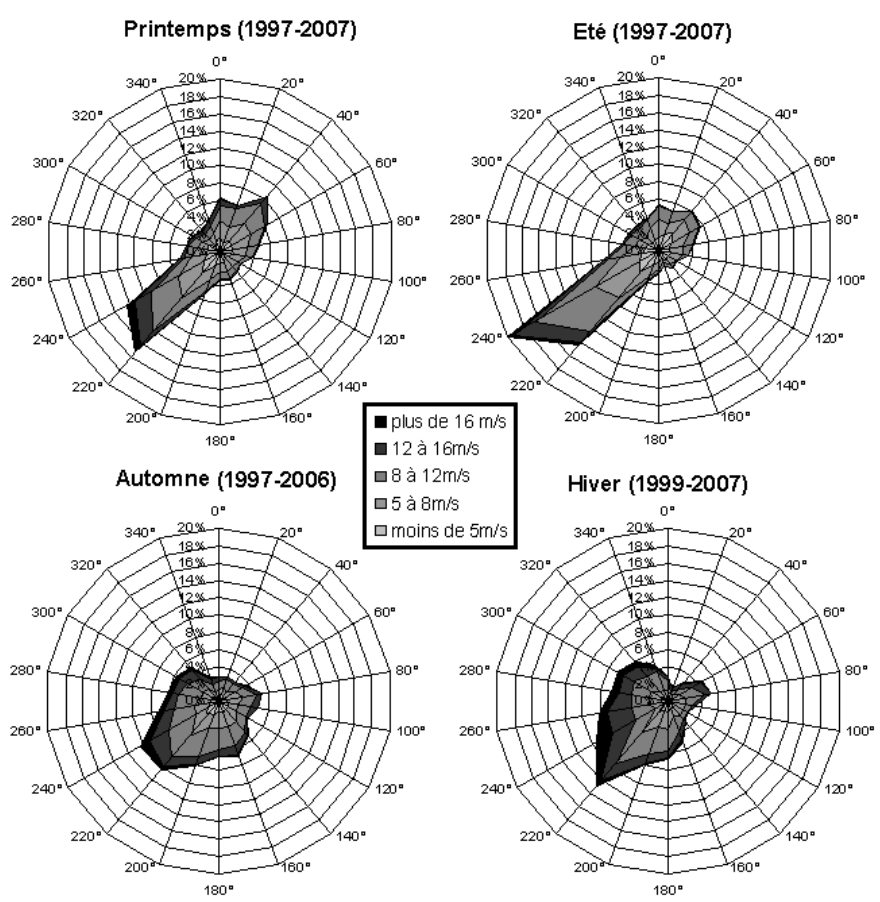

Figure 1. Roses des vents saisonnières (Données VLIZ, Westhinder, 1997-2007). 
Les vents étudiés présentent des caractéristiques saisonnières bien distinctes. Les vents en provenance du sud-ouest dominent toute l'année surtout au printemps et en été, conformément à la circulation atmosphérique générale qui se traduit par des passages dépressionnaires venant de l'Atlantique et se déplaçant d'ouest en est. On doit noter la part non négligeable des vents du quart nord-est venant de la Mer du Nord, le plus souvent observés au printemps.

L'étalement directionnel est en revanche plus fort en automne et hiver avec l'apparition de composantes nord-ouest et sud-est. Les vents faibles sont observés dans toutes les directions. Les vents les plus forts sont observés en hiver : plus de la moitié sont supérieurs à $12 \mathrm{~m} / \mathrm{s}$, les forts coups de vents $(>16 \mathrm{~m} / \mathrm{s})$ proviennent $\mathrm{du}$ large quart $220^{\circ}-320^{\circ}$ et surtout $240^{\circ}$, direction pour laquelle les rafales représentent quasiment $1 / 4$ des observations en hiver.

\section{$3 \quad$ Mise en place du modèle statistique}

\subsection{Description du modèle}

Le modèle statistique retenu est un modèle autorégressif à changement de régimes markovien d'ordre 1 de type gaussien (MSAR gaussien), décrit par l'équation :

$$
Y_{t}=N\left(a^{\left(s_{t}\right)} Y_{t-1}+b^{\left(s_{t}\right)}, \sigma^{\left(s_{t}\right)}\right)
$$

où $\left\{Y_{t}\right\}_{t=1 . . N}$ désigne la chaîne observable, soit dans notre étude la vitesse $\mathrm{du}$ vent, $\left\{S_{t}\right\}_{t=1 . . N}$ désigne la chaîne des états (ou régimes) cachés et $N(\mu, \sigma)$ la loi normale de moyenne $\mu$ et d'écart type $\sigma$.

La chaîne des états cachés $\left\{S_{t}\right\}_{t=1 . . N}$, non observable, contrôle le processus observé $\left\{Y_{t}\right\}$, comme l'illustre le graphe d'indépendance conditionnelle (Figure 2).

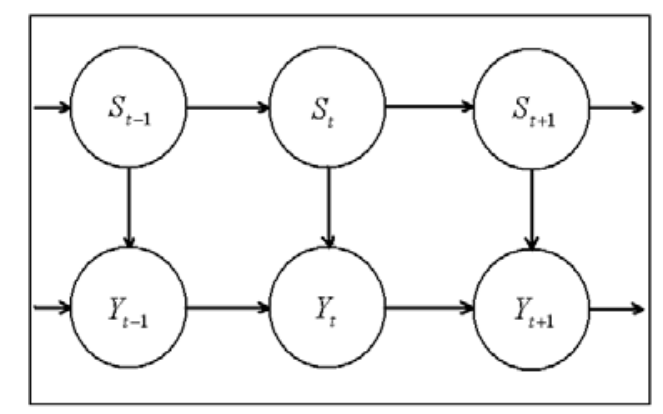

Figure 2. Graphe d'indépendance conditionnelle du modèle MSAR (D'après AILLIOT, 2004). 


\subsection{Justification du choix du modèle}

Le Bayesian Information Criterium (critère BIC) (LEBARBIER \& MARYHUARD, 2004) sélectionne le modèle à deux états cachés pour les données de vent de la bouée Westhinder.

Le modèle gaussien trouve des états correspondants à des régimes de vent pouvant être interprétés physiquement (régimes anticycloniques ou dépressionnaires, Figure 3). Cependant, la difficulté réside alors dans le fait que $Y_{t}$ peut alors prendre des valeurs négatives (équation (1)). Aussi, lors de la génération de séries temporelles, les valeurs négatives ont été rejetées, sans que cela ne dégrade sensiblement la performance du modèle. L'utilisation d'un modèle MSAR gamma, qui s'obtient en remplaçant la loi normale par une loi gamma dans l'équation (1) permettrait de contourner cette difficulté (AILLIOT, 2004).
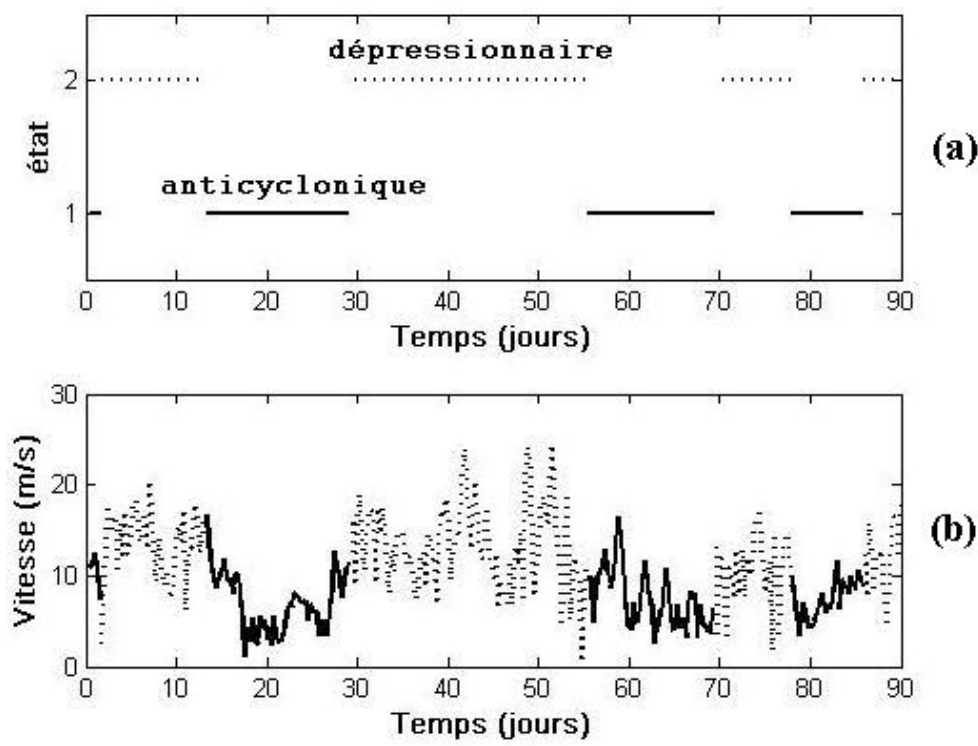

(b)

Figure 3. Identification des régimes sur l'hiver 2007 - (a) Alternance des régimes, (b) Reconnaissance des régimes sur la série temporelle.

\subsection{Calibration}

La méthode employée pour calibrer le modèle est un algorithme de type EM (Estimation/Maximisation), implémenté dans la boîte à outils METIS (MONBET \& AILLIOT, 2005). Les paramètres des lois stationnaires obtenues en appliquant l'algorithme EM aux données de la bouée Westhinder sont présentés dans le tableau 1.

L'accès à ces paramètres permet donc de générer des séries temporelles de vent 
représentatives du climat observé, en utilisant l'équation (1).

Tableau 1. Paramètres des régimes hivernaux.

\begin{tabular}{|c|c|c|c|}
\hline \multirow{2}{*}{ Régime } & \multicolumn{3}{|c|}{ Paramètres des lois stationnaires } \\
\cline { 2 - 4 } & $\begin{array}{c}\text { Moyenne } \\
(\mathbf{m} / \mathbf{s})\end{array}$ & Ecart-type (m/s) & $\begin{array}{c}\text { Temps de résidence } \\
\text { (jrs) }\end{array}$ \\
\hline "temps calme" & 7,8 & 3,5 & 3,9 \\
\hline "dépressionnaire" & 11,3 & 4,2 & 3,7 \\
\hline
\end{tabular}

\subsection{Validation}

Un certain nombre de critères statistiques ont été choisis pour tester la capacité du modèle à générer des séries statistiquement représentatives des données. 1100 séries temporelles ont été générées et leurs propriétés statistiques comparées à celles issues de la bouée, grâce à la boîte à outils METIS : histogramme, fonction d'autocorrélation, distribution des maxima annuels, durée du temps de séjour audessus et en dessous de $8 \mathrm{~m} / \mathrm{s}$ et $16.8 \mathrm{~m} / \mathrm{s}$, périodogramme. Tous ces critères se sont révélés satisfaisants et le modèle est considéré comme performant vis-à-vis des données. En voici deux exemples (Figure 4) :
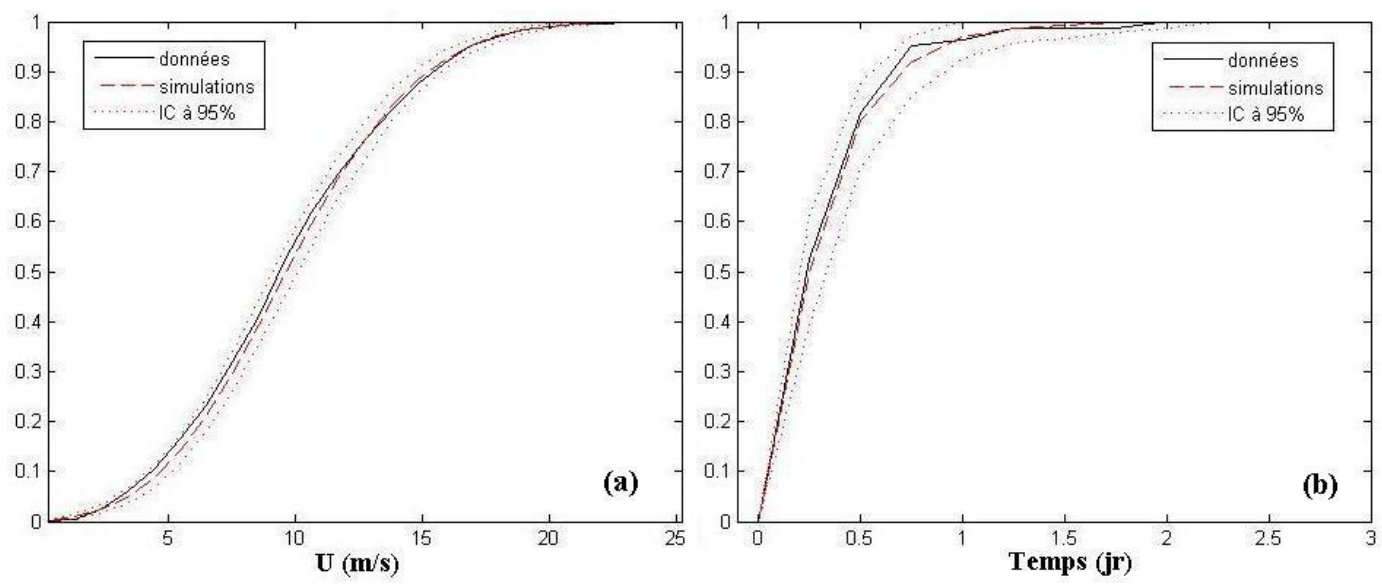

Figure 4. Exemple de critères utilisés pour évaluer la capacité du modèle à représenter les données - (a) Fonction de répartition des intensités de vent,

(b) Fonction de répartition du temps de séjour au-dessus de $16.8 \mathrm{~m} / \mathrm{s}$.

\section{$4 \quad$ Dynamique du transport éolien en zone littorale}

Le transport de sédiments par le vent est un processus morphogène important, qui est sensible à un grand nombre de paramètres, en particulier vitesse et 
direction du vent, topographie, humidité, pluie, marée et végétation (PEDREROS, 2000). Dans cette étude, nous nous limitons à l'influence de la vitesse du vent, aucun autre paramètre n'étant modélisé.

\subsection{Couche limite et mise en mouvement}

On considère un profil logarithmique suivant une loi de Von-Karman (figure 5) :

$u(z)=\frac{u_{*}}{k} \ln \left(\frac{z}{z_{0}}\right)$

où $\mathrm{u}(\mathrm{z})$ représente la vitesse moyenne du vent à l'altitude $\mathrm{z}, \mathrm{u} *$ la vitesse de friction, $\mathrm{z}_{0}$ la longueur de rugosité et $\mathrm{k}$ la constante de Von-Karman $(\mathrm{k}=0.4)$.

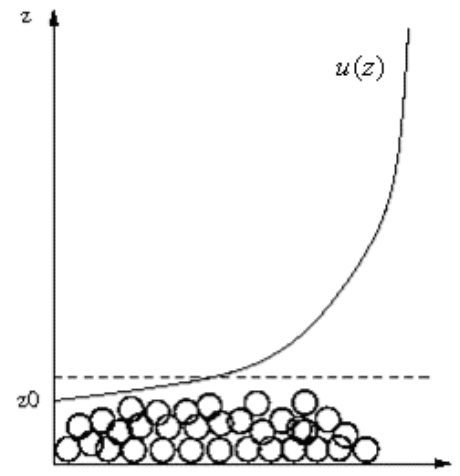

surface rugueuse

Figure 5. Profil logarithmique de l'écoulement du vent.

Les grains sont soumis à la gravité et aux forces de cohésion interparticulaires, ainsi qu'à la contrainte de cisaillement du vent. Les grains peuvent être déplacés par le vent selon trois modes : la suspension, le roulement ou la saltation. Le mode de déplacement prépondérant est la saltation, au cours de laquelle un grain éjecté par le vent retombe au sol, déclenchant ainsi la mise en mouvement d'autres grains. La mise en saltation des grains s'effectue lorsque la vitesse de friction $u *$ dépasse une vitesse de friction seuil $\mathrm{u}_{*}$. Nous avons calculé la vitesse de friction seuil $\mathrm{u}_{*_{\mathrm{t}}}$ en utilisant la formule de SHAO \& LU (2000) :

$u_{*_{t}}=\sqrt{\frac{0.0235}{\rho_{a}}\left(\frac{\pi}{6} \Delta \rho g d+\frac{0.00016}{d}\right)}$

où $\mathrm{d}$ désigne le diamètre du grain, $\Delta \rho$ la différence entre la masse volumique des grains et celle de l'air, et g l'accélération de la pesanteur. 


\subsection{Calcul du flux horizontal}

Le flux vertical des grains ne concerne que les matériaux très fins de type poussière. Sur les côtes ces grains ne sont pas rencontrés et le flux vertical est donc négligeable (PEDREROS, 2000). Le flux horizontal des grains dépend du diamètre des particules. Des formules de flux massique horizontal (c'est-à-dire la masse de particules qui traversent une section de $1 \mathrm{~m}$ linéaire de plage en $1 \mathrm{~s}$ ) classiquement rencontrées dans la littérature sont présentées dans le tableau 2.

Tableau 2. Exemples de formules de calcul du flux massique horizontal$\rho_{a}$ désigne la masse volumique de l'air, g l'accélération de la pesanteur,

$d$ le diamètre des particules et $D$ un diamètre de référence $(D=0.25 \mathrm{~mm})$, d'après PEDREROS (2000).

\begin{tabular}{|c|c|c|}
\hline Auteur & Flux horizontal $\left(\mathrm{kg} \cdot \mathrm{m}^{-1} \cdot \mathrm{s}^{-1}\right)$ & Constantes \\
\hline Bagnold (1941) & $q=B \frac{\rho_{a}}{g} \sqrt{\frac{d}{D}} u_{*}^{3}$ & $\begin{aligned} \mathrm{B}= & 1.5 \text { à } 2.8 \text { suivant } \\
& \text { le sédiment }\end{aligned}$ \\
\hline Kawamura (1951) & $q=K \frac{\rho_{a}}{g}\left(u_{*}+u_{*_{t}}\right)^{2}\left(u_{*}-u_{*_{t}}\right)$ & $\mathrm{K}=2.78$ \\
\hline $\begin{array}{l}\text { Lettau et Lettau } \\
\qquad(1978)\end{array}$ & $q=L \frac{\rho_{a}}{g}\left(1-\frac{u_{* t}}{u_{*}}\right) \sqrt{\frac{d}{D}} u_{*}^{3}$ & $\mathrm{~L}=4.2$ \\
\hline Pedreros (2000) & $q=P \frac{\rho_{a}}{g}(3.6-33.4 \sqrt{d . g})\left(u_{*}-u_{*_{t}}\right)^{2}$ & $\mathrm{P}=1.25$ \\
\hline
\end{tabular}

5 Sensibilité de l'activité de transport éolien aux paramètres du régime

\section{dépressionnaire}

Une granulométrie unique de $250 \mu \mathrm{m}$, représentative de la Baie de Wissant (RUZ \& MEUR-FEREC, 2003) a été adoptée. Nous obtenons un indicateur de l'activité du transport éolien en calculant simplement le volume de grains mis en mouvement par le vent à l'issue d'un hiver simulé.

Nous avons ensuite perturbé les paramètres du modèle statistique de manière à étudier l'impact de deux évolutions distinctes :

- le nombre de tempêtes, en modifiant la durée de résidence dans le régime dépressionnaire.

- l'intensité moyenne dans le régime dépressionnaire en modifiant le paramètre b de l'équation (1), qui est directement relié à l'intensité moyenne du vent dans ce régime. 
Dans un premier temps, pour chacun de ces paramètres, les différentes formules de transport (Tableau 2) sont testées selon une méthode de Monte-Carlo, de façon à établir une tendance générale. Dans un second temps, la variabilité intrinsèque au modèle statistique a été représentée en utilisant la formule de Pedreros (2000), avec les intervalles de confiance à 50\% et $96 \%$. Le volume des grains transportés par le vent a été calculé en faisant une moyenne sur 50 séries temporelles, pour chaque valeur du paramètre dont on teste l'influence.

\subsection{Comparaison du comportement des formules de transport}

Le régime dépressionnaire actuellement observé en hiver sur les données de la bouée Westhinder est caractérisé par une durée de $90 \mathrm{~h}$ et une vitesse moyenne de $11.3 \mathrm{~m} / \mathrm{s}$.

Les influences de ces deux paramètres ont été testées sur des intervalles allant respectivement de 0 à $180 \mathrm{~h}$ et de 0 à $22.6 \mathrm{~m} / \mathrm{s}$, ce qui correspond à un doublement des paramètres du régime observé sur les données. Les résultats de la simulation indiquent (Figures 6-a et 6-b) une influence significativement plus forte de l'intensité des tempêtes pour les 4 formules.

Pour la formule de Pedreros (2000), une augmentation de $50 \%$ de la durée moyenne du régime dépressionnaire actuel n'entraîne qu'une augmentation de $18 \%$ du volume de grains transportés (Figure 6-a). Au contraire une augmentation de $50 \%$ de l'intensité moyenne accroît le transport de 300 \% (Figure 6-b).
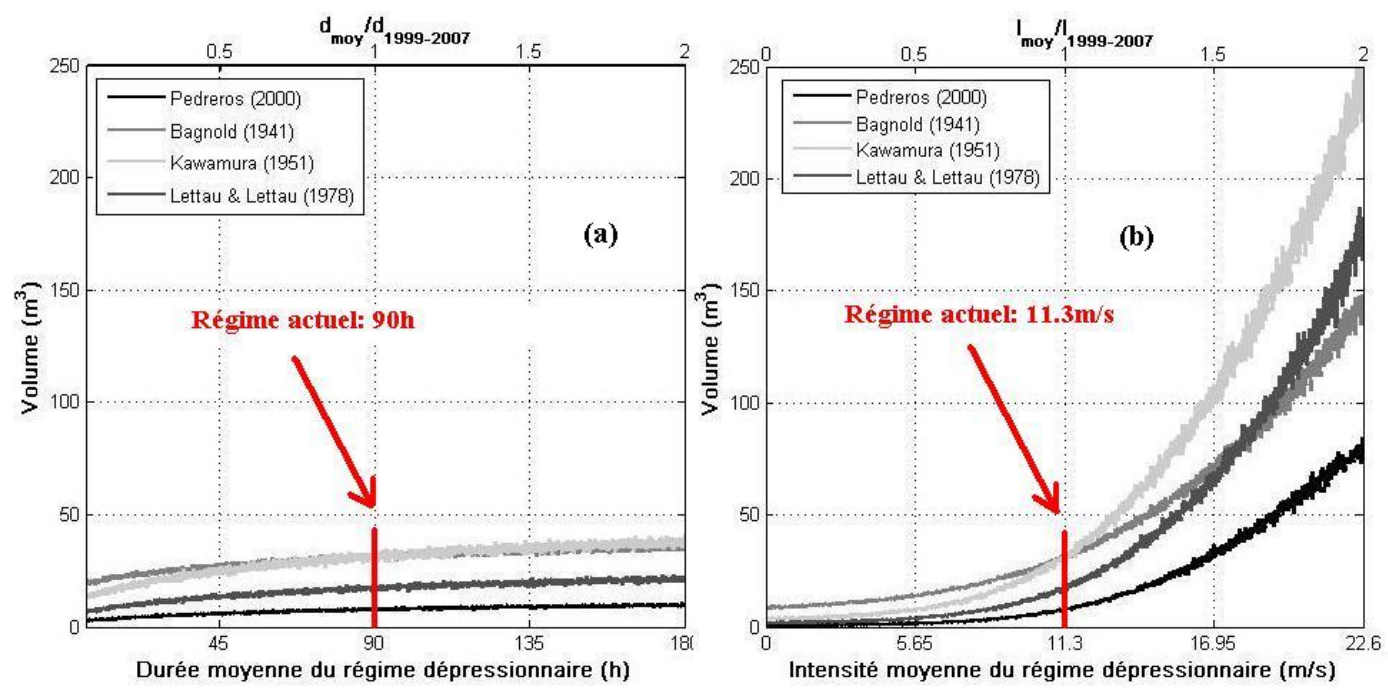

Figure 6. Influence de la durée du régime dépressionnaire (a)

et de l'intensité moyenne du régime tempête (b) sur le volume de grains transportés à l'issue de l'hiver. 


\subsection{Variabilité de l'activité de transport éolien}

La formule de Pedreros (2000), qui a été validée en milieu littoral sur la côte Aquitaine, a été choisie pour tester la variabilité du modèle, intrinsèque à son caractère statistique.
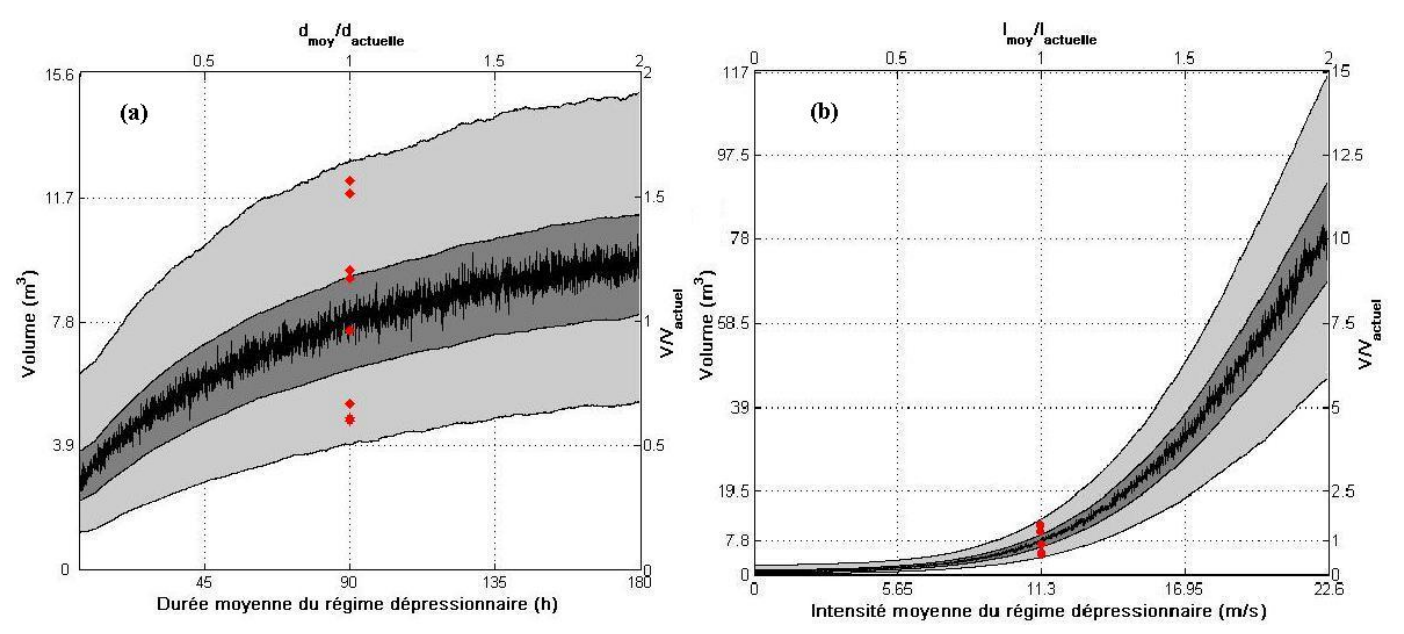

Figure 7. Variabilité du transport sous l'influence de la durée du régime dépressionnaire (a) et de l'intensité moyenne du régime dépressionnaire (b). Les ordonnées ne sont pas à la même échelle à gauche et à droite. Le transport a été calculé (points rouges) sur les séries temporelles des hivers 1999-2007 par la formule de Pedreros (2000).

Selon ces simulations, l'activité éolienne n'est plus augmentée de manière significative lorsque la durée du régime dépressionnaire croit au-delà de 15 jours. En revanche, une augmentation de l'intensité moyenne des vents dans le régime dépressionnaire accroit l'activité du transport éolien de manière toujours significative. Par ailleurs, les graphiques de la figure 7 semblent indiquer que pour une calibration donnée, la variabilité naturelle du transport éolien de sédiments est plus forte que ne le suggèrent nos simulations.

\section{Conclusions et perspectives}

$\mathrm{Au}$ cours de cette étude nous avons adopté une approche statistique pour simuler des évolutions du champ de vent à une échelle locale. Nous avons donc calibré le modèle MSAR gaussien sur des données de vent Westhinder à $30 \mathrm{~km}$ au large de Dunkerque, de façon à générer des séries temporelles de vent représentatives d'un climat local actuellement observé ou d'un climat perturbé (évolution du nombre d'épisodes de vents forts, de leur intensité). Nous avons étudié les effets de modifications des régimes de tempêtes sur le volume de 
sédiments transportés, en adoptant un modèle de transport simple sur une côte idéalisée inspirée des côtes dunaires de la région de Dunkerque. Les simulations indiquent que le transport éolien est bien plus sensible à une intensification des événements extrêmes qu'à une augmentation du nombre de ces événements.

En considérant des données à la côte et une modélisation plus fine du transport, il sera alors possible à terme d'étudier l'évolution morphodynamique d'un profil de dune réaliste en réponse à une modification du forçage par le vent.

\section{Remerciements}

Les auteurs remercient Valérie Monbet et Pierre Ailliot pour la boîte à outils METIS (Ifremer) et pour leur relecture, Arnaud Héquette (LOG) pour les données VLIZ de Westhinder, Carlos Oliveros et Déborah Idier (BRGM) pour leur relecture.

\section{Références bibliographiques}

1 AILLIOT P. (2004), Modèles autorégressifs à changements de régimes markoviens. Application aux séries temporelles de vent, Thèse, Université de Rennes I.

2 LEBARBIER E., MARY-HUARD T. (2004), Le critère BIC: fondements théoriques et interprétation, INRIA $\mathrm{n}^{\circ} 5315$.

3 LUA H. (2000), An integrated wind erosion modelling system with emphasis on dust emission and transport, $\mathrm{PhD}$, University of New South Wales, Australia.

4 MONBET V., AILliot P. (2005), Metocean Time Series (METIS) Toolbox Documentation, Ifremer.

5 MONBET V., MAISONDIEU C., LE HIR P. (2006), Evolution morphodynamique cross shore d'un estran vaseux, IXème journées nationales Génies Civil Génie Côtier, T. 2, p. 401-408.

6 PEDREROS R. (2000). Quantification et Modélisation du transport éolien au niveau des zones côtières. Application au littoral girondin, Thèse, Université de Bordeaux I.

7 RAÏSENEN J., HANSSON U., ULLERSTIG A., DÖSCHER R., GRAHAM L. P., JONES C., MEIER H. E. M., SAMUELSSON P., WILLEN U. (2003), European climate in the late $21^{\text {st }}$ century: regional simulations with two driving global models and two forcing scenarios. Rossby Centre, Swedish Meteorological and Hydrological Institute.

8 RUZ M. H., MEUR-FEREC C. (2003). Influence of high water levels on aeolian sand transport: upper beach/dune evolution on a macrotidal coast, Wissant Bay, northern France, Geomorphology 60 (2004) 73-87. 